\title{
Die Behandlung von Schlafstörungen mit Akupunktur
}

\author{
Eine Reihe von Akupunkturpunkten lässt sich bei Schlafstörungen \\ symptomatisch einsetzen - Beachtet man darüber hinaus die Konstitu- \\ tion des Patienten, hilft die Akupunktur sehr zuverlässig \\ Naschmil Pollmann
}

Schlafstörungen gehören zu den besonders erfolgsversprechenden Indikationen für die Akupunktur; selbst wenn man einfach und schematisch vorgeht; und umso mehr, wenn man die Konstitution des Patienten differenziert betrachtet.

Dieser Artikel behandelt nicht alle, aber einige der häufigsten TCM-Muster bei Schlafstörungen:

- Hitze-Erkrankungen der Leber bzw. des Herzens

- Yin-Mangel der Organe Herz, Niere und Leber

- Blut-Mangel von Herz bzw. Leber

- Nahrungsstagnation im Magen mit Entwicklung von Schleim und/oder Hitze Einige Punkte können symptomatisch bei allen Mustern eingesetzt werden (sie werden im Folgenden daher nicht jedes Mal explizit aufgeführt):

- Ex Anmian I und II (am Mastoid; 1 cun dorsal 3E 17 oder 1 cun ventral Gb 20)

- Gb 12 (eigentlich wirken alle Punkte um die Schädelbasis beruhigend, das Segment C2 ist stark vagusinnerviert)

- LG 20 Baihui mit den Sishencong (ExKH1)

- He 7

- Ma 41

- sowie am Ohr z.B. Shen Men, Polster, Jerome und Retro-Jerome, Herz u.a.

Für die Ohrakupunktur darf insgesamt ein relaxierender und vegetativ entspannender Effekt angenommen werden; sie sollte in der Behandlung von Schlafstörungen niemals fehlen [1].

\section{Schlafstörungen im Blick} der chinesischen Medizin

Körperliches und seelisches Wohlbefinden hängen davon ab, dass der Körper ausreichend mit Qi und Blut versorgt ist und das Qi im Körper frei fließen kann. Fehlt dem Körper Qi oder Yang, oder ist der freie Fluss des Qi behindert, so entstehen lethargischdepressive Zustände. Im Vordergrund stehen Adynamie und Müdigkeit, selbst wenn der eigentliche Schlaf wenig oder gar nicht gestört wird.

Fehlt dem Körper das nährende und befeuchtende Blut, kommen zu den körperlichen Symptomen des Blut-Mangels, wie z.B. Kreislaufbeschwerden, Palpitationen, (trockene) Haut, Sehstörungen, Krämpfen in der Muskulatur oder Sensibilitätsstörungen, regelhaft Schlafstörungen, Konzentrationsund Merkstörungen, Schwindel und Erschöpfung dazu.

Fehlt Yin oder überwiegt das Yang, treten Schlafstörungen, Neurasthenie und Unruhe in den Vordergrund, denn das Yin sorgt in Körper und Seele für Ausgleich und Ruhe.

Das Herz als Ort des Bewusstseins und der Wahrnehmung hat eine übergeordnete Funktion, da es als Filter und zentrale Schaltstelle dient. Nachts sollte der im Herzen ansässige denkende und koordinierende Geist Shen zu Ruhe und Erholung kommen. Das Regiment wird nun übernommen von der in der Leber wohnenden Wanderseele Hun, die für unsere Verbindung zur Welt des Un(ter)bewussten und der Ideen

\section{Zusammenfassung \\ Akupunktur ist eine empfehlens- werte Methode bei der Behand- lung von Schlafstörungen. Selbst bei schematischem Vorgehen gibt es eine Reihe symptoma- tisch einsetzbarer Akupunktur- punkte. Der Artikel stellt darü- ber hinaus die häufigsten TCM- Muster bei Schlafstörungen und die dabei auftretenden Sympto- me vor sowie die dann empfeh- lenswerten weiteren Punkte für die Therapie.}

sorgt und während des Schlafes nur lose mit dem Körper verbunden sein kann. Ein gesunder Schlaf, erholsam und nicht durch unangenehme Träume gestört, hängt entscheidend davon ab, dass Herz und Leber durch ihr Blut bzw. Yin Geist und Wanderseele ausreichend halten, nähren und verwurzeln können; sonst kann sich der Geist Shen nicht erholen und die Wanderseele Hun schweift während des Schlafes unruhig umher und bringt von ihrer Reise Albträume, Ängste und Unruhe mit.

\section{Leber-Qi-Stagnation}

Sicherlich das am häufigsten diagnostizierte TCM-Muster und die Wurzel vieler der folgenden Muster ist die Leber-Qi-Stagnation. Wer sich unter Druck fühlt, wird nicht nur Stressverarbeitung über die Muskulatur betreiben, sondern oft auch Schwierigkeiten in der Verarbeitung des am Tag Erlebten haben. Sehr häufig entsteht aus der Stauung Hitze, die nicht immer kontinuierlich vorhanden sein muss. Durchschlafstörungen ohne wesentliche Tagesmüdigkeit kommen häufig vor; gibt es - was fast die Regel ist zum gestauten Leber-Qi auch noch eine Milz-Leere, so kann das Einschlafen durch 
sich ziehen, zum einen aufgrund der besonderen Beziehungen von Herz und Niere, zum anderen da Yin und Yang der Niere die Wurzel für das Yin und Yang anderer Organe darstellen. Ein isolierter Herz-Yin-Mangel findet sich manchmal nach schweren psychischen Traumata oder nach hochfieberhaften Erkrankungen, insbesondere wenn diese den Thorax betrafen (z.B. Scharlach). Man beobachtet häufig auch die Kombination aus Blut- und Yin-Mangel, insbesondere im Klimakterium.

\section{Herz-Yin-Mangel}

Im Vordergrund stehen neben erheblichen Durchschlafstörungen mit nächtlichem Schwitzen und oft sehr ängstlich gefärbten Träumen die tiefe Erschöpfung und die seelische „Dünnhäutigkeit“. Die Patienten erwachen oft gegen 3 oder 4 Uhr, völlig nassgeschwitzt und zu Tode erschöpft, können aber nicht wieder einschlafen. Tagsüber fühlen sie sich ebenso erschöpft wie ruhelos und schreckhaft. Gedächtnis und Konzentrationsfähigkeit sind schlecht. Palpitationen und Herzrasen sowie das sog. „5 FlächenFieber" verweisen ebenso auf das Krankheitsbild wie die trockene, gerötete und an zeit der Niere) allgemeine Leere-Hitze-Zeichen, nicht nur der Nachtschweiß. Manche Patienten müssen sich mehrmals pro Nacht umziehen! Ein schwerer Nieren-Yin-Mangel findet sich als typische Schlafstörung des ängstlichen, kranken, alten Menschen, der dann auch meist noch von Rücken- und Gelenkschmerzen, Tinnitus, Schwerhörigkeit, Kontinenzproblemen und Vergesslichkeit geplagt wird. Häufig werden die Schlafstörungen noch verstärkt durch ein unangenehmes Brennen in den Fußsohlen.

\section{Therapie}

Soweit es möglich ist, das Nieren-Yin über Akupunktur anzusprechen, erfolgt dies mit den Punkten Mi6, KG4, Ni6 (Ni 1 oder 2), man wird vielleicht auch an Bl 23 oder Bl 52 denken, insbesondere bei begleitenden Rückenschmerzen.

\section{Leber-Yin-Mangel}

Der Leber-Yin-Mangel ist eine häufige Erscheinung des Klimakteriums und tritt oft kombiniert mit einem Leber-Blut-Mangel (s. u.) oder, noch häufiger, in Verbindung mit einem Nieren-Yin-Mangel auf. Ein anderer Entstehungsmodus ist der auf dem Boden

\section{Schlafen ist kein geringes Kunststück, denn man}

\section{muss den ganzen Tag dafür wach bleiben.}

\section{Friedrich Nietzsche}

der Spitze belaglose und rissige Zunge und der fadenförmige, schnelle Puls sowie ein trockener Rachen.

Man sollte hier mit eher wenig Punkten arbeiten, die für das Krankheitsbild spezifisch sind.

\section{Therapie}

- He 5, He 8, Bl 15, KG 14 oder 15 mit Bezug auf den Funktionskreis

- Mi 6, KG 4, Ni 6 (Ni 1 oder 2) als Punkte mit Wirkung auf das Yin (der Niere)

- Di 11 als den Geist beruhigender und Hitze-ableitender Punkt

\section{Nieren-Yin-Mangel}

Den Nieren-Yin-Mangel kennzeichnen neben gravierenden Einschlafstörungen und dem ebenfalls vorkommenden Aufwachen in den frühen Morgenstunden (zur Minimal- eines chronischen Leber-Feuers. Fast immer begleiten ihn Symptome des aufsteigenden Leber-Yang wie Hitzewallungen, Stimmungsschwankungen, anfallsweise Kopfschmerzen und Reizbarkeit.

\section{Therapie}

Zusätzlich zu den symptomatischen Punkten und den oben aufgeführten Punkten zur Stärkung des (Nieren)-Yin sollte auch an Le 8 (Blut- und Yin der Leber stärkend), Le 2 (entfernt Hitze aus der Leber) und $\mathrm{Bl} 18$ (Shu-Punkt) gedacht werden.

\section{Herz-Blut-Mangel}

Der Herz-Blut-Mangel ist häufig Folge einer chronischen Milz-Qi-Schwäche oder einer Disharmonie zwischen Leber und Milz. Ernährungsfehler spielen eine große Rolle; der Hang zu „gesundem Essen“ mit viel Roh- und Körner-Kost kann sich bei einer schwachen Milz als fast ebenso fatal erweisen wie eine Neigung zu Fastfood. Auch müssen Vegetarier sehr sorgfältig auf Ausgewogenheit in der Ernährung achten, da sie sonst Gefahr laufen, zumindest im chinesischen Sinne „anämisch“ zu werden. Frauen sind insgesamt häufiger betroffen als Männer; BlutMangel kann nämlich oft während oder nach Schwangerschaften entstehen, insbesondere, wenn zusätzliche Faktoren wie Überarbeitung oder Stress hinzukommen.

Auch in der Menopause kommt es häufig zu Schlafstörungen auf dem Boden von Blut (und/oder Yin)-Mangel. Die resultierende Kombination aus Schlafmangel und Vergesslichkeit ist nahezu sprichwörtlich; die „StillDemenz" ist dem Leser sicher ebenso bekannt wie der im amerikanischen Sprachraum „perimenopausal brain-fog“ genannte häufige Leistungsabfall im Klimakterium. Es gibt sowohl Ein- als auch Durchschlafstörungen, der Schlaf ist unruhig, eher leicht, man bewegt sich „zwischen Tag und Traum“. Die Träume werden als wirr und unruhig, aber oft sehr realitätsnah beschrieben. Eine gewisse Desorientierung beim Aufwachen ist häufig, seltener wird ein regelrechtes Derealisations- und Depersonalisationserleben berichtet. Die Diagnosestellung fällt leicht, schon der Blick auf die blasse, trockene Zunge ist ebenso wegweisend wie Vergesslichkeit, Schreckhaftigkeit, begleitende Palpitationen und abnorme Ermüdbarkeit.

\section{Therapie}

Neben den symptomatischen Punkten:

- Ma 36, Mi 6 (oder Mi 4) KG 6, Bl 20 stärken die Milz

- KG 14 oder 15, He 5 oder 7, Bl 15 oder 17 werden als lokale Punkte bzw. mit Funktionskreisbezug eingesetzt

\section{Leber-Blut-Mangel}

Auch der Leber-Blut-Mangel tritt besonders häufig bei Frauen auf, vor allem im Klimakterium, nach mehreren Geburten oder nach langen Phasen mit starken Blutverlusten (z.B. Hypermenorrhö), kommt aber auch konstitutionell vor. Wichtige Hinweise liefert die Zyklusanamnese (Nachlassen der Blutungsintensität, Leere-Zeichen verstärkt am Ende der Menses und in der ersten Zyklushälfte). Neben den implizierten Durchschlafstörungen und der Tagesmüdigkeit (Mittagstief!), den lebhaften und wirren Träumen gibt es einige spezifische Hinweise 
auf die Leber, wie z.B. das Schlafwandeln oder das Sprechen im Schlaf. Häufig werden Träume vom Fliegen berichtet, manchmal sogar ein regelrechtes Aus-dem-Körper-Heraustreten und über diesem Schweben, in der TCM Zeichen für eine unzureichend verwurzelte Wanderseele hun. Daneben kommen an körperlichen Symptomen Kopfschmerzen, Vergesslichkeit, Schwindel und Sehstörungen vor. trockene Haut und Schleimhäute, splitternde Nägel, diffuse Parästhesien in den Extremitäten und leider auch häufig nächtliche Wadenkrämpfe vor. Besondere Erwähnung verdient hier das Restless-Legs-Syndrom.

Das Restless-Legs-Syndrom (RLS) ist eine weit verbreitete Ursache für Schlafstörungen; ca. 5-10\% der über 50-Jährigen sind mehr oder weniger betroffen. Beschrieben werden ziehende oder kribbelnde Missempfindungen meist unterhalb der Knie, begleitet vom unwiderstehlichen Impuls, die Beine zu bewegen, was zwar eine Erleichterung bringt, den Patienten aber am (Wieder-)Einschlafen hindert. Zwar gibt es mit L-Dopa u.a. durchaus effektive Medikamente, doch werden diese nicht immer gut vertragen. Interessant ist, dass der ursächlich oder verstärkend manchmal vorhandene Folsäure- oder Eisenmangel die Brücke schlägt zum klassischen chinesischen Verständnis der Erkrankung, nämlich dem Leber-Blut- (und Yin)-Mangel mit der Entwicklung von Wind in den Leitbahnen.

\section{Therapie}

- Ma 36, Mi 6 (oder 4), KG 6, KG 12 stärken die Milz als Energielieferantin für die Blutbildung

- Pe 6, Le 8, Bl 17, Bl 18, Bl 47 (Tor der Wanderseele) als Punkte mit Funktionskreisbezug

\section{Nahrungs-Stagnation im Magen}

Die alte Regel, man solle „zu Abend essen wie ein Bettelmann“, wird häufig missachtet und hat dann die Nahrungs-Stagnation im Magen zur Folge. Durchschlafstörungen und Tagesmüdigkeit finden sich in Verbindung mit gastrointestinalen Beschwerden wie Druckgefühl im Oberbauch, leichte Übelkeit, Aufstoßen und Sodbrennen. Häufig besteht Mundgeruch; wohl auch durch den dichten Bakterienrasen auf dem pathognomonischen dicken und klebrigen Zungenbelag, der je nach dem Vorhandensein von Hitze weiß oder gelb gefärbt sein kann.
Die Störung tritt auch situativ auf. Allerdings entwickelt sich langfristig Schleim, oft mit Hitze. Der Patient hat eine ausgeprägte Tagesmüdigkeit, scheint „bleischwer“ zu schlafen, und so fühlt er sich auch am Morgen. Träume werden nur selten erinnert, es besteht das Gefühl, sich die ganze Nacht unruhig hin und her gewälzt zu haben. Während der Patient selbst den Eindruck hat, alle 5 Minuten aufgewacht zu sein, wird der Partner oder die Partnerin sich im Gegenteil darüber beklagen, selbst nicht schlafen zu können, und zwar wegen des lautstarken Schnarchens im Bett. Hier empfiehlt sich meist die Untersuchung hinsichtlich einer Schlafapnoe.

\section{Therapie}

Neben ordnungstherapeutischen Hinweisen behandelt man das Grundmuster mit:

- Ma 36, Mi 6, KG 6 und KG 12 stärken das Qi von Milz und Magen und helfen so, die Stagnation zu beseitigen

- Ma 40, 41, 44 entfernen Schleim und Hitze aus dem Magen

- Pe 6 hilft gegen Übelkeit, Di 11 beruhigt den Geist und entfernt Hitze

\section{Schleim-Hitze des Herzens}

Bei besonders ungeordneten Patienten mit neben den Schlafstörungen auch ausgeprägten Persönlichkeitsveränderungen wird man manchmal auf die Schleim-Hitze des Herzens stoßen. Das Krankheitsbild kann sich auf dem Boden eines Leber-Feuers entwickeln, aber auch seinen Ausgang in einer Magenerkrankung nehmen (Nahrungsstagnation im Magen, Magen-Feuer). Vielfach geht Alkohol-, Drogen- oder Medikamentenmissbrauch voraus. Neben Unruhe, Schlaflosigkeit oft über Tage, Albträumen, Palpitationen und thorakalem Völlegefühl zeigt sich das Vorhandensein von Schleim durch Schwindel, Benommenheitsgefühl, Konzentrations- und Gedächtnisstörungen sowie einen zunehmenden Verlust der klaren Urteilsfähigkeit. Auf der Zunge findet sich neben den oben erwähnten Rötungen oder Ulzerationen ein dicker, klebriger gelber oder brauner Zungenbelag.

\section{Therapie}

Neben den Punkten, die bereits beim Herz-

Feuer erwähnt wurden:

- Ma 40, 41, Di 11

- Pe 5 oder 6

- KG 22
Fazit

Insgesamt ist die Akupunktur eine durchaus empfehlenswerte Methode bei der Behandlung von Schlafstörungen, insbesondere dann, wenn man den Patienten noch einige einfache ordnungstherapeutische Regeln an die Hand gibt:

- Das Meiden von zu reichlichem Essen,

- zu aufregenden Krimis und

- zu vielen Zigaretten vor dem Schlafengehen.

Interessenkonflikt: Die Autorin erklärt, dass keine wirtschaftlichen oder persönlichen Verbindungen bestehen.

Online zu finden unter

http://dx.doi.org/10.1055/s-0034-1386737

\section{Literatur \\ 1 Chen HY, Shi Y, Ng CS, Chan SM, Yung KK, Zhang QL. Auricular acupuncture treatment for insomnia: a systematic review. J Altern Comple- ment Med. 2007; 13 (6): 669-676}

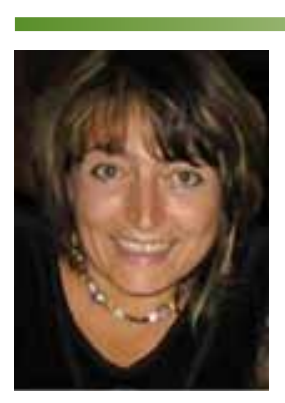

Naschmil Pollmann

Praxis für Schmerztherapie und Naturheilverfahren Blankenese Erik-Blumenfeld-Platz 21

22587 Hamburg Naschmil@googlemail.com

Naschmil Pollmann ist Ärztin für Anästhesiologie, Spezielle Schmerztherapie, Naturheilverfahren/Umweltmedizin. Sie ist seit fast 20 Jahren als Schmerztherapeutin niedergelassen und arbeitet in ihrer Praxis vorwiegend mit Akupunktur und chinesischer Arzneitherapie sowie Osteopathie. Sie ist seit 1997 als Dozentin bei der DÄGfA tätig und Autorin des „Kursbuch Akupunktur“. 